This item was submitted to Loughborough's Research Repository by the author.

Items in Figshare are protected by copyright, with all rights reserved, unless otherwise indicated.

\title{
Transformation of a shoaling undular bore
}

PLEASE CITE THE PUBLISHED VERSION

http://dx.doi.org/10.1017/jfm.2012.338

PUBLISHER

(c) Cambridge University Press

VERSION

AM (Accepted Manuscript)

LICENCE

CC BY-NC-ND 4.0

REPOSITORY RECORD

El, G.A., Roger H.J. Grimshaw, and Wei K. Tiong. 2019. "Transformation of a Shoaling Undular Bore". figshare. https://hdl.handle.net/2134/15334. 
This item was submitted to Loughborough's Institutional Repository (https://dspace.lboro.ac.uk/) by the author and is made available under the following Creative Commons Licence conditions.

\section{creative
commons}

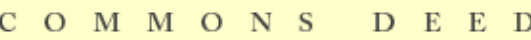

Attribution-NonCommercial-NoDerivs 2.5

You are free:

- to copy, distribute, display, and perform the work

Under the following conditions:

Attribution. You must attribute the work in the manner specified b the author or licensor.

Noncommercial. You may not use this work for commercial purposes.

No Derivative Works. You may not alter, transform, or build upon this work.

- For any reuse or distribution, you must make clear to others the license terms of this work.

- Any of these conditions can be waived if you get permission from the copyright holder.

Your fair use and other rights are in no way affected by the above.

This is a human-readable summary of the Leqal Code (the full license).

\section{Disclaimer 만}

For the full text of this licence, please go to: http://creativecommons.org/licenses/by-nc-nd/2.5/ 


\title{
Transformation of a shoaling undular bore
}

\author{
G.A. El, R.H.J. Grimshaw and W.K. Tiong \\ Department of Mathematical Sciences, Loughborough University, \\ Loughborough LE11 3TU, UK
}

(Received )

We consider the propagation of a shallow-water undular bore over a gentle monotonic bottom slope connecting two regions of constant depth, in the framework of the variablecoefficient Korteweg - de Vries equation. We show that, when the undular bore advances in the direction of decreasing depth, its interaction with the slowly varying topography results, apart from an adiabatic deformation of the bore itself, in the generation of a sequence of isolated solitons - an expanding large-amplitude modulated solitary wavetrain propagating ahead of the bore. Using nonlinear modulation theory we construct an asymptotic solution describing the formation and evolution of this solitary wavetrain. Our analytical solution is supported by direct numerical simulations. The presented analysis can be extended to other systems describing the propagation of undular bores (dispersive shock waves) in weakly non-uniform environments.

\section{Introduction}

Description of the interaction of a shallow water wave with variable topography is a classical and fundamental problem of fluid mechanics. This problem has been thoroughly studied for linear waves (see, e.g. Johnson (1997) and references therein) as well as for isolated solitary waves (see e.g. Grimshaw (2007a) and references therein). Much less is known about the propagation of nonlinear wavetrains over obstacles. In spite of being of considerable interest for applications, in particular, in coastal oceanography (see e.g. 
Scotti et al. (2008), Tissier et al. (2011) and references therein) this problem remains almost unexplored theoretically except for very special model cases where exact analytic solutions are available (see Ostrovsky \& Pelinovsky (1975), Miles (1983a), and Grimshaw $(2007 a))$

Nonlinear wavetrains are often generated in the form of unsteady undular bores connecting two different basic flow states and exhibiting solitary waves at one of the edges. Undular bores are usually formed as a result of dispersive resolution of a shock or an initial discontinuity in fluid depth/velocity (see e.g. Smyth \& Holloway (1988), El et al. (2006), Esler \& Pearce (2011)) or due to the resonant interaction of a fluid flow with variable topography (see e.g. Grimshaw \& Smyth (1986), Baines (1995), El et al. (2009)).

In the framework of a weakly nonlinear long wave paradigm, the propagation of a shallow-water undular bore over a flat bottom, in the absence of dissipation effects, is asymptotically described by the slowly modulated periodic solution of the constantcoefficient $\mathrm{KdV}$ equation. The evolution of the modulation parameters (such as mean value, amplitude, wavenumber etc.) in the undular bore is then described by an expansion fan solution of the Whitham averaged equations (Gurevich \& Pitaevskii (1974), Fornberg \& Whitham (1978)).

In the presence of variable topography, the relevant model for the weakly nonlinear long-wave propagation is the variable-coefficient $\mathrm{KdV}$ equation which, for slow topography variations can be asymptotically reduced to the perturbed constant-coefficient KdV equation. The corresponding perturbed modulation equations were derived in El et al. (2007). They take into account variations of the undular bore parameters due to varying environment and bottom friction, in addition to the modulations resulting from spatial non-uniformity of the initial or boundary conditions. Analysis of the behaviour of the characteristics of the modulation system for the perturbed KdV equation in the vicinity 
of the leading edge of the undular bore in El et al. (2007) has enabled one to identify two possible scenarios of the undular bore evolution with respect to the behaviour of its leading solitary wave:

(i) "Weak interaction" (local) scenario. In this scenario, the undular bore propagates so that its lead soliton evolves as an isolated, adiabatically varying solitary wave;

(ii) "Strong interaction" (nonlocal) scenario. The undular bore evolves so that its lead soliton evolution is determined not only by the local variations of the topography or/and the presence of bottom friction but also by the interaction with the entire nonlinear wavetrain behind it.

The actual scenario of the propagation of an undular bore over a slope depends on the slope polarity and on the relative values of the characteristic scales in the problem (the typical slope value, the bottom friction coefficient, the typical wavelength in the bore and the typical spatial scale for modulations).

In this paper, we further explore the undular bore on a slope problem by considering in detail a special case when the undular bore propagation occurs over a broad but finite region of decreasing depth. Such a configuration is relevant to the modelling of the near-shore tsunami propagation (see e.g. Madsen et al. (2008), Tissier et al. (2011) and references therein) but is also of a broader significance in the context of the general description of the dispersive shock wave propagation in weakly inhomogeneous media.

The main result of the paper is that, if the undular bore advances in the direction of decreasing depth, its interaction with the slowly varying topography results in the formation of a sequence of isolated solitons - an expanding modulated solitary wavetrain attached to and propagating ahead of the bore. Importantly, this solitary wavetrain is not part of the bore and its generation constitutes a non-adiabatic response of the undular bore to the slow topography variations, which is not captured by the classical Gurevich- 
Pitaevskii type solutions assumed in El et al. (2007). Here we construct an appropriate extension of the traditional undular bore modulation theory to include an asymptotic description of the generation and evolution of the advancing solitary wavetrains. Our analytical solution is supported by direct numerical simulations.

\section{Problem formulation}

We start with the canonical model for a weakly nonlinear unidirectional shallow-water wave propagation over uneven bottom, which is the variable-coefficient KdV equation (Kawahara (1975), see also Grimshaw (2007b) and references therein)

$$
A_{t}+c A_{x}+\frac{c_{x}}{2} A+\frac{3 c}{2 h} A A_{x}+\frac{c h^{2}}{6} A_{x x x}=0 .
$$

This is written here in non-dimensional form, based on a length scale $h_{0}$, a representative

depth, and a time scale $\sqrt{h_{0} / g}$. Here $A(x, t)$ is the free surface elevation above the undisturbed non-dimensional depth $h(x)$, while $c(x)=\sqrt{h(x)}$ is the non-dimensional linear long wave phase speed. The variable-coefficient $\mathrm{KdV}$ equation in the form asymptotically equivalent to (2.1) was first systematically derived for surface gravity waves by Kakutani (1971) and Johnson (1973), and an analogous equation for internal waves by Grimshaw (1981). The first two terms in (2.1) are the dominant terms, and by themselves describe the propagation of a linear long wave with speed $c$. The remaining terms represent, respectively, the effect of varying depth, weakly nonlinear effects and weak dispersion. The $\mathrm{KdV}$ equation for constant depth can be derived as an asymptotic shallow-water smallamplitude reduction of the full Euler system for irrotational flows using the usual balance in which $\partial / \partial t \sim \partial / \partial x \sim \epsilon \ll 1, A \sim \epsilon^{2}$ (see, e.g., Johnson (1997)). In the derivation of (2.1) weak inhomogeneity is added to this balance so that $c_{x} / c$ scales as $\epsilon^{3}$. For simplicity we ignore here the term describing effects of bottom friction (see e.g. Miles (1983b) or El et al. (2007)), this could be introduced later. 
We can cast (2.1) into the asymptotically equivalent form

$$
\begin{aligned}
& A_{\tau}+\frac{h_{\tau}}{4 h} A+\frac{3}{2 h} A A_{X}+\frac{h}{6} A_{X X X}=0, \\
& \text { where } \quad \tau=\int_{0}^{x} \frac{d x^{\prime}}{c\left(x^{\prime}\right)}, \quad X=\tau-t .
\end{aligned}
$$

Here $h=h(\tau)$ explicitly depends on the variable $\tau$ which describes evolution along the path of the wave. Formally we write $A(x, t)=\tilde{A}(X, \tau)$ and $h(x)=\tilde{h}(\tau)$ but then omit the "tilde" in (2.2). The balance of terms in (2.2) is ensured by $\partial / \partial \tau \sim \epsilon^{3}, \partial / \partial X \sim \epsilon$, $A \sim \epsilon^{2}$. Thus, unlike in the original variable-coefficient $\mathrm{KdV}$ equation (2.1), where both independent variables $x$ and $t$ vary on the same scale $\sim 1 / \epsilon$, in $(2.2)$ the "time" $\tau$ is a slow variable relative to the "spatial" coordinate $X$. We stress that equations (2.1) and (2.2) are asymptotically equivalent: they differ with respect to terms of $O\left(\epsilon^{7}\right)$, which is the same as the error term in both equations.

We shall suppose that

$$
h(x)=1 \text { for } x<0, \quad h(x)=h_{1} \text { for } x>x_{1},
$$

and varies monotonically in $0 \leq x \leq x_{1}$. We assume that $x_{1} \gg 1$. Then for times $t<0$ we shall suppose that we have an initial condition imposed in $x<0$, that is

$$
A(x, t=0)=A_{0}(x) \text { for } x<x_{0}<0, \quad A(x, t=0)=0 \text { for } x>x_{0} .
$$

Thus initially we generate a solution of the constant coefficient KdV equation, and the aim is to see how this develops in $x>0$. The special case when $A_{0}$ is a constant for $x<x_{0}$ generates an undular bore. In this paper we study the propagation of an undular bore over a decreasing depth region, i.e. $h_{1}<1$. The setting under consideration is illustrated in Figure 1. In terms of the "signalling" variables (2.3) the initial condition (2.5) becomes

$$
\tilde{A}(X(t=0)=\tau, \tau)=A_{0}(x),
$$

where $x(\tau)$ is given by (2.3). However, because $\tau$ is a slow variable relative to $X$, we have 


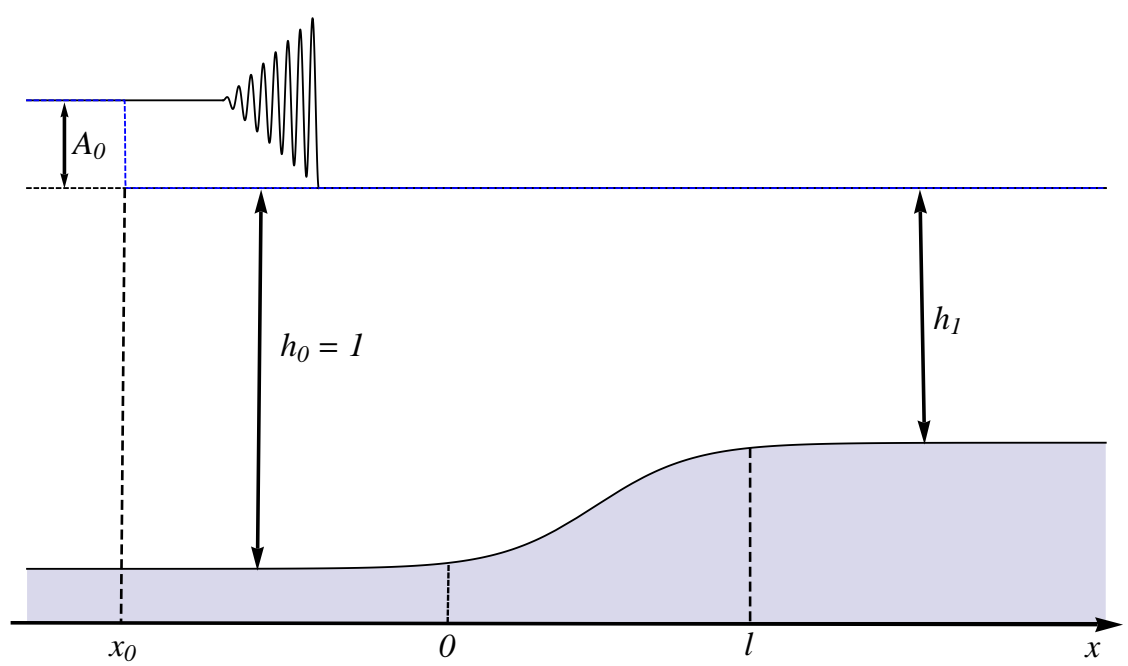

FiguRE 1. Propagation of an undular bore over sloping bottom: problem setting

$\tilde{A}(X, \tau)=\tilde{A}(X, 0)+O\left(\epsilon^{3}\right)$ so asymptotically, the initial condition for (2.2) is

$$
A(X, \tau=0)=A_{0}(X),
$$

where we have again omitted the "tilde" for $A$ and used the fact that $A_{0}(x)$ is only non-zero in $x<0$ where $\tau=x$, and so $X=x$ at $t=0$.

The governing equation (2.2) can be cast into several equivalent forms. That most commonly used is the variable-coefficient KdV equation, obtained here by putting

$$
B=h^{1 / 4} A, \quad \text { so that } \quad B_{\tau}+\frac{3}{2 h^{5 / 4}} B B_{X}+\frac{h}{6} B_{X X X}=0
$$

This form shows that equation (2.2) has two integrals of motion with the densities proportional to $B=h^{1 / 4} A$ and $B^{2}=h^{1 / 2} A^{2}$. These are often referred to as laws for the conservation of "mass" and "momentum".

It is often convenient to recast (2.2) into the standard $\mathrm{KdV}$ equation form with constant coefficients, modified by a certain perturbation terms. Thus we introduce the new variables (see e.g. Newell (1985) or El et al. (2007))

$$
u=\frac{3}{2 h^{2}} A=\frac{3}{2 h^{9 / 4}} B, \quad S=\frac{1}{6} \int_{0}^{\tau} h\left(\tau^{\prime}\right) d \tau^{\prime}=\frac{1}{6} \int_{0}^{x} h\left(x^{\prime}\right)^{1 / 2} d x^{\prime} .
$$




$$
\text { so that } u_{S}+6 u u_{X}+u_{X X X}=-\frac{9 h_{S}}{4 h} u \text {. }
$$

In this form, the governing equation (2.10) has the structure of the integrable $\mathrm{KdV}$ equation on the left-hand side, while the effect of varying depth is on the right-hand side. This structure enables one to use the modulation theory for perturbed integrable systems developed in Kamchatnov (2004). Yet another convenient form for (2.2) is obtained by putting

$$
\begin{gathered}
T=\frac{1}{6} \int_{0}^{\tau} \frac{d \tau^{\prime}}{h^{5 / 4}\left(\tau^{\prime}\right)}=\frac{1}{6} \int_{0}^{x} \frac{d x^{\prime}}{h^{7 / 4}\left(x^{\prime}\right)} \\
U=\frac{3 B}{2} \quad \text { so that } \quad U_{T}+6 U U_{X}+\beta(T) U_{X X X}=0 \quad \text { where } \quad \beta(T)=h^{9 / 4} .
\end{gathered}
$$

In this formulation we assume that $\beta(T)=1$ for $0<T<T_{0}$ and $\beta(T)=\beta_{1}$ for $T>T_{1}$ with a monotonic variation in $T_{0} \leq T \leq T_{1}$. Note that the equations $(2.2,2.8,2.10,2.12)$ are exactly equivalent. The initial condition for $(2.10,2.12)$ are

$$
u(X, S=0)=u_{0}(X)=U(X, T=0)=U_{0}(X)=\frac{3}{2} A_{0}(X) .
$$

Note that in all the equations $(2.2,2.8,2.10,2.12)$ we shall refer to $\tau, S, T$ respectively as "time", although they are in fact a variable describing evolution along the path of the wave.

\section{Undular bore transformation over the slope}

When the bottom is flat, so that $\beta=1$ in (2.12), we assume that the initial condition is that $U(X, T=0)=H(-X) U_{0}, U_{0}=3 A_{0} / 2$. Then the decay of the initial discontinuity at $X=0$ leads to the development of an undular bore, an expanding slowly modulated periodic wavetrain, asymptotically described by the Whitham modulation theory. The 
local wave form of the undular bore is given by the periodic KdV solution

$$
\begin{aligned}
\quad U & =a\left\{b(m)+\mathrm{cn}^{2}\left(\frac{q}{\beta^{1 / 2}}\left(X-X_{0}-V T\right) ; m\right)\right\}+d, \\
\text { where } \quad b & =\frac{1-m}{m}-\frac{E(m)}{m K(m)}, \quad a=2 m q^{2}, \\
\text { and } \quad V & =6 d+2 a\left\{\frac{2-m}{m}-\frac{3 E(m)}{m K(m)}\right\} .
\end{aligned}
$$

Here $\operatorname{cn}(x ; m)$ is the Jacobi elliptic function of modulus $m(0<m<1)$ and $K(m), E(m)$ are the elliptic integrals of the first and second kind respectively, $a$ is the wave amplitude, $d$ is the mean level, $V$ is the wave speed, and $X_{0}$ is a constant defining the initial phase. Note that we have retained $\beta$ in (3.1) and (3.3), in order to include the case when $\beta \neq 1$ on the shelf. Also, if the characteristic scale for slow variations of $\beta$ with time is much greater than that of the modulation parameters in the undular bore, expression (3.1) remains asymptotically valid for $\beta_{T} \neq 0$. The value $\beta^{1 / 2} / q$ can be identified as a measure of the width of the wave humps while their spatial period (wavelength) is

$$
L=\frac{2 K(m) \beta^{1 / 2}}{q}
$$

Note that for $1-m \ll 1$, we have $L \gg \beta^{1 / 2} / q$. This family of solutions contains three free parameters, which are chosen from the set $\{a, q, V, d, m\}$. As $m \rightarrow 1, \operatorname{cn}(x ; m) \rightarrow \operatorname{sech}(x)$ and then the cnoidal wave (3.1) becomes a solitary wave, riding on a background level $d$. On the other hand, as $m \rightarrow 0, \operatorname{cn}(x ; m) \rightarrow \cos x$ and so the cnoidal wave (3.1) collapses to a linear sinusoidal wave (note that in this limit $a \rightarrow 0$ ).

The Whitham modulation theory assumes that the expression (3.1) describes a modulated wave in which the amplitude $a$, the mean level $d$, the speed $V$ and the modulus $m$ are all slowly varying functions of $X$ and $T$. The outcome is a set of three nonlinear hyperbolic equations for three of the available free parameters, chosen from the set $(a, q, V, d, m)$, or rather better, from an appropriate combination of them. These equations are often called the Whitham equations. 
The relevant asymptotic solution is then constructed in terms of the similarity variable $X / T$, and is given by

$$
\begin{aligned}
& \frac{X}{T}=2 U_{0}\left\{1+m-\frac{2 m(1-m) K(m)}{E(m)-(1-m) K(m)}\right\} \\
& \quad \text { for } \quad-6 U_{0}<\frac{X}{T}<4 U_{0}, \\
& a=2 U_{0} m, \quad d=U_{0}\left\{m-1+\frac{2 E(m)}{K(m)}\right\}, \quad q=U_{0}{ }^{1 / 2} .
\end{aligned}
$$

Note that this solution does not depend on the value of $\beta$, the latter affects only the wavelength of the underlying periodic wave (3.1). The wavenumber distribution in the undular bore is then given by

$$
k=\frac{2 \pi}{L}=\frac{\pi U_{0}^{1 / 2}}{\beta^{1 / 2} K(m)} .
$$

Ahead of the wavetrain where $X / T>4 U_{0}, U=0$ and at this end, $m \rightarrow 1, a \rightarrow 2 U_{0}$ and $d \rightarrow 0$; the leading wave is a solitary wave of amplitude $2 U_{0}$ relative to a mean level of 0 . Behind the wavetrain where $X / T<-6 U_{0}, U=U_{0}$ and at this end $m \rightarrow 0, a \rightarrow 0$, and $d \rightarrow U_{0}$; the wavetrain is now sinusoidal with the wavelength $L=\pi\left(\beta / U_{0}\right)^{1 / 2}$, which holds throughout the wavetrain, so all waves behind the undular bore have the same spatial wavelength (for $\beta=$ const, otherwise the wavelength slowly varies with $T$ ).

The solution (3.4), (3.5) is due to Gurevich \& Pitaevskii (1974) (see also Fornberg \& Whitham (1978)), where it was obtained in terms of the Riemann invariants of the Whitham modulation equations (Whitham (1965)). It is important to note that the leading solitary wave in the undular bore developing from an initial step represents asymptotically, as $T \rightarrow \infty$, a genuine isolated $\mathrm{KdV}$ soliton, not constrained by the interactions with the remainder of the bore (see Khruslov (1976) or more recent investigation by Claeys \& Grava (2010)). We also note that the asymptotic behaviour of the wavenumber $k$ (3.6) near the leading edge $X=X_{+}$implied by solution (3.4), (3.5) is $k \sim 1 / \ln (1 / \delta)$, where $\delta=\left(X_{+}-X\right) /\left(X_{+}-X_{-}\right) \ll 1, X_{-}$being the trailing edge of the undular bore (see 
Gurevich \& Pitaevskii (1974), El (2005)). This behaviour can be viewed as a "threshold" between the "weak interaction" and "strong interaction" scenarios arising in the consideration of the propagation of undular bores over variable topographies (see El et al. (2007)): if $k \lesssim 1 / \ln (1 / \delta)$ then the solitary wave interactions near the leading edge are weak, and the lead solitary wave of the undular bore behaves as an isolated soliton; however, if $k \gg 1 / \ln (1 / \delta)$ near the leading edge, then the propagation of the lead solitary wave is strongly affected by its interaction with the remainder of the wavetrain.

If $U_{0}<0$ in the initial condition (3.1), then an "undular bore" solution analogous to that described by $(3.1,3.4)$ does not exist. Instead, the asymptotic solution is a rarefraction wave,

$$
\begin{aligned}
& U=0 \quad \text { for } \quad X>0, \\
& U=-\frac{X}{6 T} \quad \text { for } \quad 6 U_{0}<\frac{X}{T}<0, \\
& U=U_{0}, \quad \text { for } \quad \frac{X}{T}<6 U_{0}(<0) .
\end{aligned}
$$

Small oscillatory wavetrains are needed to smooth out the discontinuities in $U_{X}$ at the corners.

We concentrate here on the case $U_{0}>0$ producing an undular bore. If the slope is sufficiently gentle, one could expect that the undular bore would undergo some adiabatic change during the interaction with varying topography while retaining its structure as a slowly modulated nonlinear periodic wavetrain with a soliton at the leading edge and the linear vanishing amplitude wavepacket at the trailing edge. This initial assumption that the interaction with the topography does not change the structure of the bore has an immediate implication that the amplitude of the leading soliton for $T>T_{1}$ has the same value $a=2 U_{0}$ as in the initial bore.

Indeed, one can readily infer from the KdV equation (2.12) that the jump $[U]$ across the 
whole propagating nonlinear wave structure is unaffected by the depth variations. Let the structure be confined to some interval $X_{a}(T)<X<X_{b}(T)$ so that $[U]=U\left(X_{b}\right)-U\left(X_{a}\right)$. Then it follows from (2.12) that $[U]_{T}=\frac{\partial}{\partial T} \int_{X_{a}}^{X_{b}} U_{X} d X=0$ provided $U_{X}=U_{X X X}=0$ at $X=X_{a, b}(T)$. Thus, since the wavetrain advances into the undisturbed depth region, $U\left(X_{b}\right)=0$, one has

$$
[U]=-U_{0} \quad \text { for all } \quad T>0
$$

Note that this result is not only unaffected by the varying coefficient $\beta(T)$ but is also independent of the actual form of the structure. Now, if it is assumed that a single undular bore emerges onto the shelf with $\beta=\beta_{1}$ then (3.8) implies that relevant modulation solution for $T>T_{1}$ will have the same form (3.4) but with $X$ generally replaced by $X-\chi(m)$, where $\chi(m)$ is some function, since, due to the presence of the variable region, the modulation solution cannot remain a centred fan but must become a more general, simple-wave solution of the Whitham equations. As a matter of fact, the form of the function $\chi(m)$ depends on the variable coefficient $\beta(T)$ and can be found only from the full solution of the perturbed Whitham equations derived in El et al. (2007). Further, the constant initial phase $X_{0}$ entering the travelling wave solution (3.1) also should become the function $X_{0}(m)$ in the transformed undular bore and can be viewed as a "modulation phase shift" acquired by the undular bore due to its interaction with variable topography. We note that a similar phase shift arises in the interactions of dispersive shock waves with rarefaction waves (Ablowitz et al. (2009); El et al. (2011)), which is to some degree analogous to the present problem of the transformation of the undular over sloping bottom (see the end of this Section). Importantly, the mentioned phase shifts do not affect relationships (3.5) between the modulation parameters in the periodic solution, which implies that amplitude of the leading solitary wave of the transformed bore would then be $2 U_{0}$, that is, unchanged from the value before the variable depth region is encountered. 
However, if one now accepts that the leading solitary wave in the undular bore evolves over a slope as an isolated soliton (the "weak interaction scenario", an assumption to be confirmed in Section 6 by the analysis of the behaviour of the modulation Riemann invariants near the leading edge of the undular bore), its amplitude must vary adiabatically, $a=2 U_{0} \beta^{-1 / 3}$ to conserve the action flux $\int U^{2} d X$ (see Section 4), so that for $T>T_{1}$ the leading solitary wave amplitude is $2 U_{0} \beta_{1}^{-1 / 3}>2 U_{0}$ for the decreasing depth profile, which is clearly inconsistent with the described above evolution scenario based on the assumption of a single undular bore emerging onto the shelf. To resolve the above inconsistency an additional solitary wavetrain at the front of the undular bore is needed to provide the gradual increase of the amplitude from $2 U_{0}$ at the undular bore leading edge to the value $2 U_{0} \beta_{1}^{-1 / 3}$ implied by the action flux conservation for an isolated soliton.

Thus, the propagation of an undular bore over a broad region of slowly decreasing depth leads to a non-adiabatic effect, the generation of a solitary wavetrain in front of the bore. The adiabatic deformation of the bore itself is twofold: (i) the change of the characteristic scale of the oscillations in the bore due to the change of the dispersion coefficient $\beta$ in (2.12); (ii) the occurrence of the additional slow 'modulation phase shift' $X_{0}(m)$ throughout the bore so that the relevant modulation solution generally represents a non-centred simple wave of the Whitham equations.

The outlined undular bore transformation can be clearly seen in Figure 2 (right panel) where we present the results of the numerical simulations of the original variablecoefficient KdV equation (2.1) with the following equilibrium depth profile

$$
h(x)=\left\{\begin{array}{lr}
1 & x<0, \\
1-\alpha x & 0<x<400, \\
0.64 & x>400 .
\end{array}\right.
$$

The initial condition for $(2.1)$ was taken in the form $A(x, 0)=\frac{1}{5}(1-\tanh (x+400))$ so that 
the wave breaking and formation of an undular bore occurs for $x<0$. For comparison, the left panel of Figure 2 presents the evolution of the undular bore generated by the same initial condition but propagating over flat bottom, $h(x)=1$. We need to stress that the plots in Figure 2 are made primarily for the illustration purposes as they provide only a qualitative picture of the evolution of a shoaling shallow-water undular bore. Indeed, the initial surface elevation jump value 0.4 assumed in the numerical simulations is way beyond the range of validity of the variable-coefficient $\mathrm{KdV}$ equation (2.1) in the present context of shallow-water surface gravity waves. Such a large value of the initial jump was chosen to decrease the (quite significant) computational time necessary for the undular bore to reach the stage when it entirely propagates in the shelf region after the sloping segment. The undular bores generated by smaller initial steps (within the range of applicability of the $\mathrm{KdV}$ equation) require much longer numerical time to reveal the same salient features of the propagation over slowly varying topography.

Another point to be made here is that the numerical solutions of equation (2.1) presented in Figure 2 show the evolution in physical $(x, t)$-space, while all our subsequent analyses will be made for the asymptotically equivalent forms (2.12) or (2.8) of the variable-coefficient $\mathrm{KdV}$ equations, in which the role of the evolution variable is essentially played by the coordinate $x$, while the "retarded" time $X=\tau-t$ plays the role of the spatial variable. These latter modifications of the $\mathrm{KdV}$ equation are necessary for the analytical progress to be made possible, although the results they provide for undular bores are not always have an intuitively transparent form. Having this in mind, we have undertaken numerical simulations of the decay of an initial discontinuity for the variable-coefficient $\mathrm{KdV}$ equation in the form (2.12) to see that all the qualitative features of the undular bore transformation observed in Figure 2 are preserved under the transition to the asymptotically equivalent formulations of the problem $(2.12),(2.8)$, 
(2.6). The detailed analysis of our simulations will be presented in Section 5; here (see Figure 3 ) we only show the boundaries $X_{ \pm}(T)$ of the undular bore along with the leading edge $X_{s}(T)$ of the solitary wavetrain. The plot in Figure 3 corresponds to the numerical solution of the $\mathrm{KdV}$ equation (2.12) with the variable coefficient $\beta(T)$ in the form of a very smooth transition between $\beta=1$ and $\beta \approx 0.367$ over the interval $\Delta T \approx 1300$, and the initial condition in the form of a step $U(X, 0)=0.5 H(-X)$.

Along with the generation of the advancing solitary wave train, this plot reveals another feature of the undular bore propagation on a slope, which is not obvious from the plots of the oscillating wave field in Figure 2 and is related to the dynamics of the trailing edge $X_{-}(T)$. One can observe a non-monotonic behaviour of the curve $X_{-}(T)$ in the interval $300 \lesssim T \lesssim 3000$, which apparently is related to the occurrence of the earlier mentioned spatial shift $\chi(m)$ in the modulation solution (3.5) leading to the "de-centring" of the expansion fan. This results, for large $T$, in the stationary shift for the curve $X_{-}(T)$ relative to its initial behaviour. One can also observe the multiphase dynamics occurring in the rear part of the bore while it propagates over the sloping region. The multiphase behaviour continues for some time after the bore emerges onto the shelf (cf. plot 3 in the right panel of Figure 2) and, at sufficiently large times, $T \gtrsim 3000$ the singlephase slowly modulated wave behaviour throughout the whole wavetrain restores. The described transient multiphase dynamics near the trailing edge does not affect the front, "soliton", part of the bore which retains its regular single-phase structure at all times.

One can clearly see the confirmation of the three main conclusions following from our analysis so far: (i) the propagation of the shallow water undular bore into the region of slowly decreasing depth leads to the generation of the large-amplitude solitary wavetrain ahead of the bore; (ii) the undular bore edge speeds, $X_{ \pm}^{\prime}(T)$, asymptotically restore their original values $X_{-}^{\prime}(T)=\cot \left(\theta_{1}\right)$ and $X_{+}^{\prime}=\cot \left(\theta_{2}\right)$ upon the emergence of the bore onto 
the shelf at $T>T_{1}$; (iii) there are spatial shifts in the positions of the transformed undular bore edges $X_{ \pm}(T)$ relative to those that would have taken place in the original bore in the absence of the variable topography. 

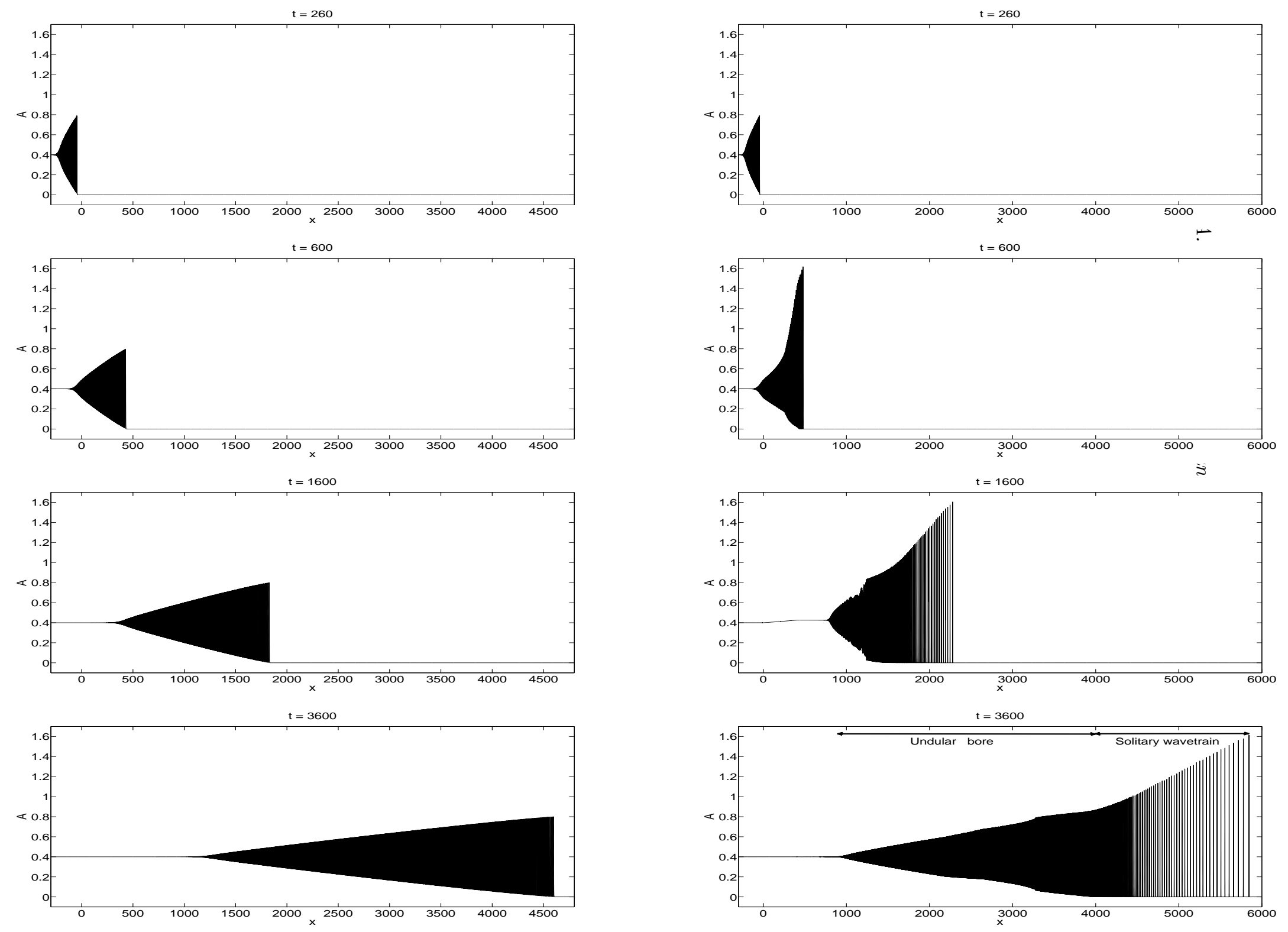

FIGURE 2. Numerical solution of the variable-coefficient KdV equation (2.1) with the initial condition $A(x, 0)=\frac{1}{5}(1-\tanh (x+400))$. Left panel: an undular bore propagating over a flat bottom $h(x)=1$; Right panel: propagation of the same (initially) undular bore 


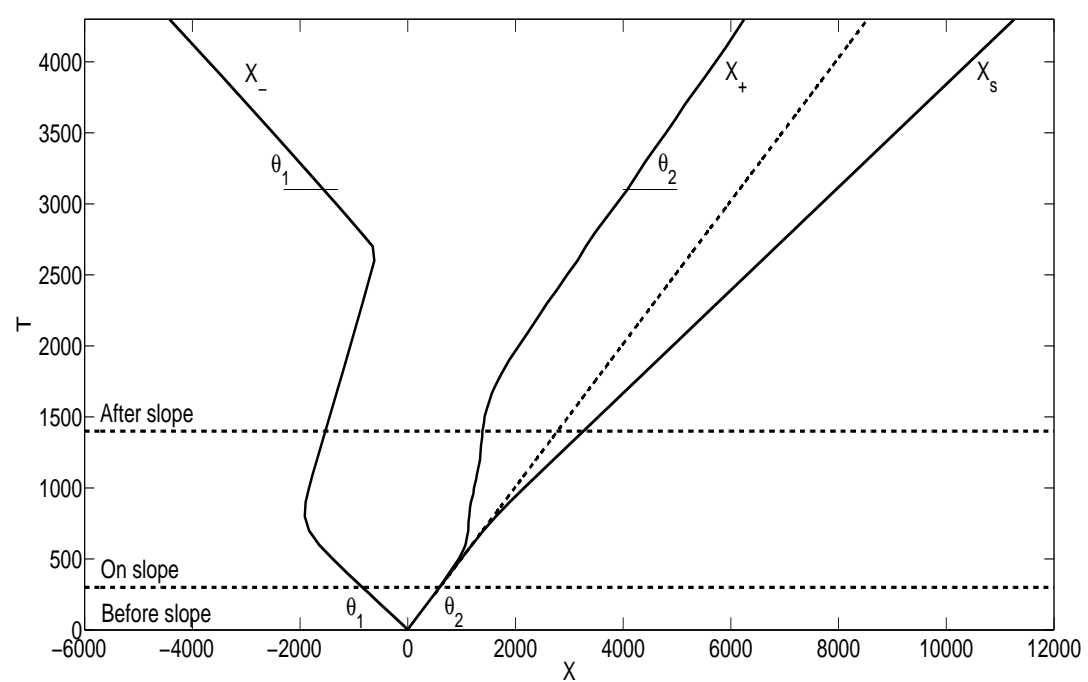

Figure 3. $X-T$ plane of the evolution of an undular bore according to the KdV equation (2.12) with variable dispersion coefficient $\beta(T)=h^{9 / 4}(T)$ where $h(T)=\frac{9}{50}\left(1-\tanh \left(\frac{T-450}{150}\right)\right)+0.64$ and the initial condition $U(X, 0)=0.5 H(-X)$ : numerical simulation data. The undular bore and the solitary wavetrain are confined to $\left[X_{-}(T), X_{+}(T)\right]$ and $\left[X_{+}(T), X_{s}(T)\right]$ respectively. The dashed line shows an extrapolation of the leading edge $X_{+}(T)$ of the initial undular bore so one can see the spatial shift of $X_{+}(T)$ due to the interaction with the slope.

It is interesting to note that the generation of an advancing soliton train in front of the undular bore was recently observed in Ablowitz et al. (2009) for one of the cases of the overtaking interaction of a dispersive shock wave with a rarefaction wave studied in the framework of the constant-coefficient $\mathrm{KdV}$ equation. Another interesting feature seen in the corresponding numerical plots in the Ablowitz et al. (2009) paper is the presence of a transient multi-phase behaviour for the undular bore interacting with the rarefaction wave. This behaviour can also be clearly seen in Figure 2 (plot 3 in the right panel). These similarities between two apparently different problems are not surprising when one observes that, in our present formulation the undular bore essentially propagates through the "rarefaction region" of decreasing depth. The essential difference is, of course, that 
in our problem the profile $h(x)$ of the "rarefaction region" is fixed and described by variable coefficients in the $\mathrm{KdV}$ equation while the evolving profile of the hydrodynamic rarefaction wave in Ablowitz et al. (2009) is given by the relevant solution (3.7) of the constant-coefficient KdV equation.

\section{Formation and evolution of the solitary wavetrain}

In this section we construct an asymptotic description of the solitary wavetrain forming in front of the undular bore that advances into the decreasing depth region. We start with the standard results for the adiabatic evolution of a single soliton in a slowly varying environment and then proceed with the analysis of the sequence of solitary waves attached to the leading edge of the undular bore.

It is well-known (Boussinesq (1872)) that a solitary wave propagating over a slowly varying bottom will deform adiabatically so that its amplitude varies as $h^{-1}$. To demonstrate this it is best to use (2.2), (2.8) or (2.12) as these have the conservation law for wave action flux. Thus, for instance,

$$
\frac{d}{d T} \int_{-\infty}^{\infty} U^{2} d X=0
$$

The slowly varying solitary wave for (2.12) is

$$
U \sim a \operatorname{sech}^{2}\{\gamma(X-\Phi(T))\}, \quad V=\Phi_{T}=2 a=4 \beta \gamma^{2},
$$

where the amplitude $a$ etc. are slowly varying function of $T$. Substitution into (4.1) gives

$$
\frac{a^{2}}{\gamma}=2 \beta^{2} \gamma^{3}=\text { constant }
$$

and so $\gamma \sim \beta^{-2 / 3}$ and $a \sim \beta^{-1 / 3}$. Thus, since $\beta=h^{9 / 4}$, we have $a \sim h^{-3 / 4}$ and noting that $U \sim h^{1 / 4} A$, the result follows. The leading wave in the undular bore is a solitary wave whose amplitude for $0<T<T_{0}$ is $2 U_{0}$. We will assume, as the undular bore propagates and deforms over the slope, this leading solitary wave will behave as if detached from 
the undular bore (the 'weak interaction scenario', El et al. (2007) and Section 5 below). Then its amplitude $a=2 U_{0} \beta^{-1 / 3}$ and it will emerge onto the shelf region $T>T_{1}$ with an amplitude $2 U_{0} \beta_{1}^{-1 / 3}$. Its trajectory is

$$
X=\Phi(T)=\int_{0}^{T} 4 U_{0} \beta\left(T^{\prime}\right)^{-1 / 3} d T^{\prime}
$$

and it reaches $T=T_{1}$ at $X=X_{1}=\Phi\left(T_{1}\right)$.

More generally, a slowly-varying solitary wavetrain propagating over a zero background can be constructed as a modulated periodic "lattice" of solitary waves (4.2) with a large spatial period so that the solitons overlap only in the regions of their tails, where the functions are exponentially small, and thus can be regarded as non-interacting. We note that in fact, a periodic lattice of solitons $\sim \sum_{n \in \mathbb{Z}} \operatorname{sech}^{2}(\lambda(x+n L))$, where $\lambda$ is the inverse "half-width", converges pointwise to the cnoidal wave solution (3.1) for any period $L>0$ (see e.g. Whitham (1984)). The condition $L \gg \lambda^{-1}$ for such a soliton lattice is equivalent to the condition $1-m \ll 1$ in the more conventional representation of the periodic solution (3.1) in terms of the Jacobi elliptic function).

Thus, locally, the solitary wavetrain can be described, up to a constant phase shift, by an asymptotic expression

$$
\begin{gathered}
0<X<1 / \kappa: \quad U \sim a \operatorname{sech}^{2}(\gamma \Theta), \quad \Theta_{T}=-\kappa V, \quad \Theta_{X}=\kappa \\
\text { where } \quad V=2 a=4 \beta \gamma^{2} \kappa^{2} \\
U(\Theta+1)=U(\Theta) .
\end{gathered}
$$

The term "locally" here implies distances comparable with a single spatial period $L=$ $1 / \kappa$. In a modulated solitary wavetrain, $a$ and $\kappa$ are slowly varying functions of $X$ and $T$ (i.e. they vary on the scale much larger than $1 / \kappa$ ) so that $(4.5)$ can be viewed as a natural extension of (4.2) for a single slowly varying soliton, and it reduces (within each period) to that expression when one sets $\kappa=1$. The fact that the initial phase is not 
fixed in (4.5) is not essential for our consideration as we shall be interested only in the behaviour of slow modulations $a(X, T), \kappa(X, T)$. Also note that expression (4.5) agrees with (3.1) in the limit when $m \rightarrow 1$ if one assumes $q=\kappa \gamma \beta^{1 / 2}$.

The modulation equations for the amplitude $a$ and the soliton train wavenumber $\kappa$ then follow:

$$
\begin{array}{r}
\left\{\frac{a^{2}}{\kappa \gamma}\right\}_{T}+V\left\{\frac{a^{2}}{\kappa \gamma}\right\}_{X}=0 \\
\kappa_{T}+(V \kappa)_{X}=0 .
\end{array}
$$

The system (4.7), (4.8) was obtained by Grimshaw (1979) (see the zero mean, $A=0$, reduction of equation (2.19)) using a multiple-scale expansion of the variable-coefficient $\mathrm{KdV}$ solution with the leading term in the form (4.5). The amplitude equation (4.7) can also be obtained directly, using averaging of the $\mathrm{KdV}$ "mass" conservation law over the periodic soliton lattice (4.5) or via the averaged Lagrangian approach (Whitham (1974)). It can also be shown to be consistent with the soliton $(m \rightarrow 1)$ limit of the full Whitham modulation system derived in El et al. (2007) for the perturbed KdV equation (2.10). It should be stressed that, while the amplitude equation (4.7) represents an exact reduction of the full modulation system where one sets $m=1$, equation (4.8) is valid only asymptotically for $1-m \ll 1$ i.e within the range of validity of the approximation $(4.5)$.

Using the relations (4.5), equations (4.7), (4.8) can be written in the form

$$
\begin{aligned}
\mathcal{A}_{\sigma}+2 \mathcal{A} \mathcal{A}_{X} & =0, \quad \mathcal{A}=\left\{\frac{a^{2}}{\sqrt{2} \kappa \gamma}\right\}^{2 / 3}=a \beta^{1 / 3} \\
\kappa_{\sigma}+(2 \mathcal{A} \kappa)_{X} & =0 \\
\text { where } \sigma & =\int_{0}^{T} \beta\left(T^{\prime}\right)^{-1 / 3} d T^{\prime} .
\end{aligned}
$$

Remarkably, the system $(4.9,4.10)$ for $\mathcal{A}(X, \sigma)$ and $\kappa(X, \sigma)$ has the same form as system 
$(4.7,4.8)$ for $a(X, T)$ and $\kappa(X, T)$ in the case when $\beta=\beta_{0}=$ constant (and so $a=$ $\left.4 \beta_{0} \gamma^{2} \kappa^{2}\right)$, i.e. for the constant-coefficient KdV equation - see Whitham (1974), Ch. 16 .

When there is no $X$-variation, $\mathcal{A}, \kappa$ are constants, and the result (4.3) is recovered. The general solution of $(4.9,4.10)$ is found by using characteristics,

$$
\begin{aligned}
& \mathcal{A}=\text { constant }, \quad \text { on } \quad \frac{d X}{d \sigma}=2 \mathcal{A}, \\
& \text { and } \frac{d \kappa}{d \sigma}=-2 \mathcal{A}_{X} \kappa=\frac{\mathcal{A}_{\sigma}}{\mathcal{A}} \kappa .
\end{aligned}
$$

Note that the system (4.12), (4.13) has only one multiple characteristic family and all the characteristics are straight lines in the $X-\sigma$ plane.

We define the position of the trailing edge of the solitary wavetrain as the line $X=$ $X_{+}(T)$ where $a=2 U_{0}$. This definition is consistent with the location of the leading edge of the undular bore for the flat bottom propagation case, and initially $X_{+}^{\prime}\left(T_{0}\right)=4 U_{0}$. However, for the varying bottom case the line $X=X_{+}(T)$ is not associated with the trajectory of a particular solitary wave as the solitary waves must be allowed to cross this boundary to enable the formation of the advancing modulated solitary wavetrain; therefore

$$
0<X_{+}^{\prime}(T)<4 U_{0} \quad \text { for } \quad T_{0}<T<T_{1} \text {. }
$$

Thus, the following boundary condition must be satisfied for the solitary wavetrain amplitude equation (4.9),

$$
\mathcal{A}=2 U_{0} \beta^{1 / 3} \quad \text { on } \quad X=\bar{X}(\sigma)
$$

where $\bar{X}(\sigma)=X_{+}(T(\sigma)) ; T(\sigma)$ being the inverse relation of (4.11), so that $\sigma(T(\sigma))=\sigma$. The latter relationship requires that $\beta(T)$ varies monotonically from 1 at $T=T_{0}$ to $\beta_{1}$ at $T=T_{1}$.

The solution for $\mathcal{A}$ is

$$
\mathcal{A}=\mathcal{A}_{0}\left(\sigma_{0}\right)=2 U_{0} \beta^{1 / 3}\left(T\left(\sigma_{0}\right)\right), \quad X-\bar{X}\left(\sigma_{0}\right)=2 \mathcal{A}_{0} \cdot\left(\sigma-\sigma_{0}\right)
$$


$\sigma_{0} \in\left[0, \sigma_{1}\right]$ being a parameter on the curve $X=\bar{X}(\sigma)$. Formally, elimination of the parameter $\sigma_{0}$ from (4.16) yields $\mathcal{A}$ as a function of $X, \sigma$. The solution (4.16) is defined for $\bar{X}(\sigma)<X<X_{s}$, where $X_{s}=4 U_{0} \sigma$ is the trajectory of the leading soliton in the solitary wavetrain (see (4.4)), having the amplitude $a=2 U_{0} \beta^{-1 / 3}$, i.e. $\mathcal{A}=2 U_{0}$. For $X>4 U_{0} \sigma$ we have $\mathcal{A}=0$. Calculating the derivative $\mathcal{A}_{X}$ we obtain:

$$
\mathcal{A}_{X}=\frac{\mathcal{A}_{0}^{\prime}\left(\sigma_{0}\right)}{2 \mathcal{A}_{0}^{\prime}\left(\sigma_{0}\right)\left(\sigma-\sigma_{0}\right)+\left[\bar{X}^{\prime}\left(\sigma_{0}\right)-2 \mathcal{A}_{0}\left(\sigma_{0}\right)\right]} .
$$

Owing to (4.14) $\left[\bar{X}^{\prime}\left(\sigma_{0}\right)-2 \mathcal{A}_{0}\left(\sigma_{0}\right)\right]<0$, therefore to guarantee the existence of the obtained solution for all $X, \sigma$ one must have $\mathcal{A}_{0}^{\prime}<0$. This, by (4.16), (4.11) implies $\beta^{\prime}(T)<0$. Then our solution represents a rarefaction fan emanating from the curve $X=\bar{X}\left(\sigma_{0}\right)$. The condition $\beta^{\prime}(T)<0$ (decreasing depth) thus can be viewed as the condition of the formation of an expanding solitary wavetrain in front of the bore. One can say that the decreasing depth 'promotes' the detachment of solitary waves at the leading edge of the undular bore. This also confirms our initial assumption that the leading solitary wave of the undular bore behaves as an isolated KdV soliton when the bore advances into decreasing depth region. A direct numerical confirmation of this assumption will be presented in Section 5 .

Using $A(X, \sigma)$ defined by (4.16) the corresponding general solution for $\kappa$ is then found from (4.13), that is

$$
\kappa=\kappa_{0}\left\{1+\frac{2 \mathcal{A}_{0}^{\prime}\left(\sigma_{0}\right)\left(\sigma-\sigma_{0}\right)}{\bar{X}^{\prime}\left(\sigma_{0}\right)-2 \mathcal{A}_{0}\left(\sigma_{0}\right)}\right\}^{-1} .
$$

where $\kappa_{0}$ is the value of $\kappa$ on the curve $X=X_{+}\left(T\left(\sigma_{0}\right)\right)=\bar{X}\left(\sigma_{0}\right)$ and $\sigma_{0}(X, \sigma)$ is defined by (4.16).

Generally, to find the curve $X=X_{+}(T)$ for $T_{0}<T<T_{1}$ one needs to solve the full perturbed modulation system from El et al. (2007). However, it is instructive to assume 
that

$$
X_{+}^{\prime}(T) \ll 4 U_{0} \quad \text { for } \quad T_{0}<T<T_{1},
$$

and thus $\bar{X}^{\prime}\left(\sigma_{0}\right) \ll 2 \mathcal{A}_{0}\left(\sigma_{0}\right)$. This behaviour can be formally justified for functions $\beta(T)$ varying sufficiently fast on a typical time scale of the solitary wavetrain modulations (but still being slow functions on the time scale of a single soliton). Indeed, since $X_{+}^{\prime}\left(T_{0}\right)=$ $4 U_{0}$, in order to satisfy $(4.14)$ one must have $X_{+}^{\prime \prime}(T)<0$ ( it is clear that $\operatorname{sign} X_{+}^{\prime \prime}(T)=$ $\operatorname{sign} \beta^{\prime}(T)$ ), then the result follows as an asymptotic behaviour of $X_{+}(T)$ for sufficiently large $T$. Thus we have

$$
X_{+}(T) \simeq X_{+}\left(T_{0}\right)=4 U_{0} T_{0} \quad \text { for } \quad T_{0}<T<T_{1} .
$$

Our numerical simulations show that behaviour (4.20) establishes itself quite quickly even for rather slow functions $\beta(T)$. One can see this in the numerical plot corresponding to $\beta_{T} \sim 5 \cdot 10^{-4}$ shown in Figure 3. For larger values of $\beta_{T}$, say $\beta_{T} \sim 10^{-3}-10^{-2}$, the boundary $X_{+}(T)$ becomes stationary almost immediately as variations of $\beta$ begin at $T=T_{0} ; X_{+}$resumes its motion at $T=T_{1}$ and gradually restores its initial velocity $4 U_{0}$. Thus (4.19) and (4.20) can be safely used in the solution (4.18), (4.16) for a broad range of the slope values specified in terms of $\beta(T)$. The schematic behaviour of the boundaries $X_{+}(T)$ and $X_{s}(T)$ illustrating the asymptotic formulation of the problem of the generation of the solitary wavetrain on the given boundary $X=X_{+}(T)=X_{+}\left(T_{0}\right)$ for $T_{0}<T<T_{1}$ is shown on Figure 4. Thus, using (4.19) we have to leading order

$$
\kappa \simeq \kappa_{0}\left\{1-\frac{\mathcal{A}_{0}^{\prime}\left(\sigma_{0}\right)\left(\sigma-\sigma_{0}\right)}{\mathcal{A}_{0}\left(\sigma_{0}\right)}\right\}^{-1}=\kappa_{0}\left\{1-\frac{2}{3} \frac{\beta^{\prime}\left(\sigma_{0}\right)}{\beta\left(\sigma_{0}\right)}\left(\sigma-\sigma_{0}\right)\right\}^{-1},
$$

where $\beta\left(\sigma_{0}\right) \equiv \beta\left(T\left(\sigma_{0}\right)\right)$ so that $\beta^{\prime}\left(\sigma_{0}\right)=\beta_{T} \beta^{1 / 3}\left(\sigma_{0}\right)<0$ and therefore solution (4.21) exists for all $X, \sigma$. Then the leading edge of the undular bore, that is also the trailing edge of the solitary wavetrain, emerging on the shelf is $X_{+}(T) \simeq 4 U_{0} T_{0}+4 U_{0}\left(T-T_{1}\right)$ for $T>T_{1}$ and the phase shift $\Delta_{+}=\chi(1)$ (see Section 3 for the definition of $\chi(m)$ ) can 


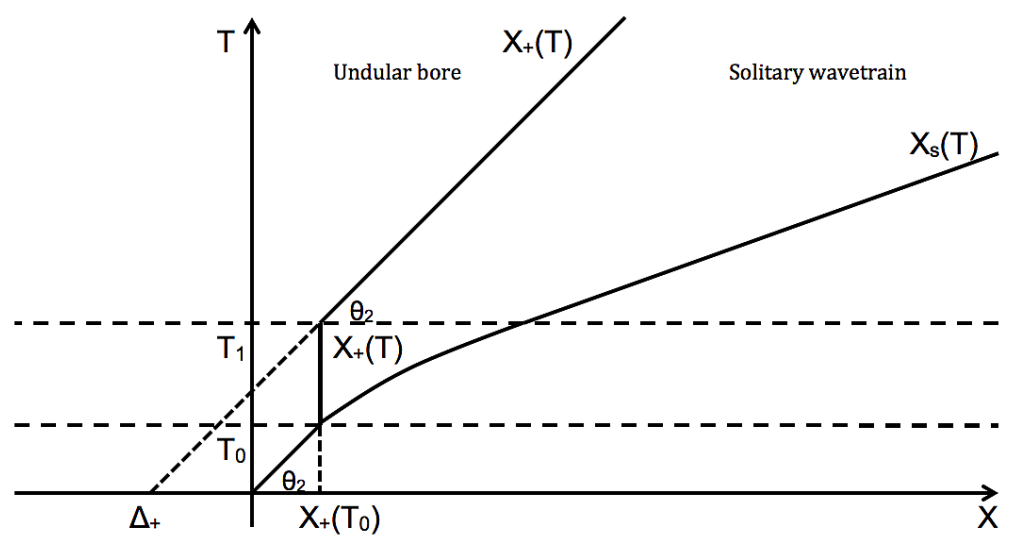

Figure 4 . Schematic behaviour of the boundaries $X_{+}$and $X_{s}$ of the solitary wavetrain generated at the leading edge of the undular bore on a slope.

be estimated as $\Delta_{+} \simeq-4 U_{0}\left(T_{1}-T_{0}\right)$. Of course, one can guarantee the linear behaviour of $X_{+}(T) \sim 4 U_{0} T$ only for $T \gg T_{1}$ when the slowly modulated structure of the undular bore fully adjusts itself to the shelf region with $\beta=\beta_{1}$.

Let $\sigma_{1}=\sigma\left(T_{1}\right)$. Then on the shelf where $T>T_{1}, \sigma>\sigma_{1}$ we have $\beta=\beta_{1}, \sigma=$ $\sigma_{1}+\left(T-T_{1}\right) \beta_{1}^{-1 / 3}$. The leading edge of the solitary wavetrain on the shelf is $X_{s}=$ $4 U_{0} \sigma=4 U_{0}\left(\sigma_{1}+\left(T-T_{1}\right) \beta_{1}^{-1 / 3}\right)$. We note that for $T>T_{1}$ both both boundaries $X_{+}(T)$ and $X_{s}(T)$ confining the expansion fan are characteristics and the total number of solitary waves in the train for $T>T_{1}$ does not change with time. The value

$$
\kappa_{0}\left(\sigma_{0}\right) \simeq \frac{U_{0}^{1 / 2}}{4 \beta^{1 / 2}\left(\sigma_{0}\right)} I, \quad \text { where } \quad I \approx 0.6569,
$$

is found by examining the undular bore structure near its leading edge (see Appendix).

Now, from (4.6), (4.9) we have $\gamma(X, T)=\kappa^{-1} \beta^{-1 / 3}(\mathcal{A} / 2)^{1 / 2}$ and so the slowly varying solitary wavetrain (4.5) is fully defined. As $T \rightarrow \infty, \sigma \sim T \beta_{1}^{-1 / 3}, X_{+} \sim 4 U_{0} T, X_{s} \sim$ $4 U_{0} \beta_{1}^{-1 / 3} T$ and the asymptotic solution is,

$$
\begin{gathered}
4 U_{0} T<X<4 U_{0} \beta_{1}^{-1 / 3} T: \quad \mathcal{A} \sim \frac{X}{2 \sigma}, \quad \text { or } \quad a \sim \frac{X}{2 T}, \\
\kappa \sim \frac{g(X /(2 T))}{\sigma} \sim \frac{g(\mathcal{A})}{\sigma}, \quad \text { or } \quad \kappa \sim \frac{G(a)}{T} .
\end{gathered}
$$


Here $g(\mathcal{A})=3 \kappa_{0} \beta\left(\sigma_{0}\right) /\left(2 \beta^{\prime}\left(\sigma_{0}\right)\right)$, where $\sigma_{0}(\mathcal{A})$ is found from the solution $\mathcal{A}=2 U_{0} \beta^{1 / 3}\left(\sigma_{0}\right)$ (see (4.16)). As a matter of fact, $g(\mathcal{A})$ is only defined for the variable coefficient region $0<\sigma_{0}<\sigma_{1}$, where $\beta^{\prime}(\sigma) \neq 0$ and where the generation of the solitary wavetrain occurs. Note that the asymptotic behaviour of the wavenumber (4.24) in the solitary wavetrain is markedly different from that in the undular bore in the vicinity of the leading edge (see formula (6.3) in the Appendix).

The function $G(a)=g\left(a \beta_{1}^{1 / 3}\right)$ in (4.24) has the meaning of the distribution function over amplitude in the solitary wavetrain so that $G(a) d a$ is the number of solitons with amplitudes in the interval $[a, a+d a]$ (see Whitham 1974). Since the total number of solitons $N$ in the train remains constant for $T>T_{1}$ it can be estimated by the formula

$$
N \simeq \int_{2 U_{0}}^{2 U_{0} \beta_{1}^{-1 / 3}} G(a) d a .
$$

In concluding this section we note that if $\beta^{\prime}(T)>0$ (increasing depth) our solution (4.21) yields that the interaction of the solitary wavetrain with the increasing depth bottom profile topography would result in an increase of the density of solitary waves enhancing thus their interaction and eventually rendering invalid the basic assumption about the isolated character of solitary waves in the train. Also, the amplitude profile specified by the solution (4.16) with $\beta^{\prime}(T)>0$ will develop a breaking singularity at some $\sigma=\sigma_{c}$ also making this solution physically invalid. All this suggests that one should not expect the generation of the chain of individual non-interacting solitons in front of the bore if $\beta^{\prime}(T)>0$. Instead, this case appears to be relevant to the realisation of the second (nonlocal) "strong interaction" scenario of the undular bore evolution discussed in El et al. (2007) and outlined in the Introduction. We plan to study this case in our future publications.

The obtained description of the transformation of an undular bore over a slope is most readily illustrated using a diagram showing the behaviour of the Riemann invariants 

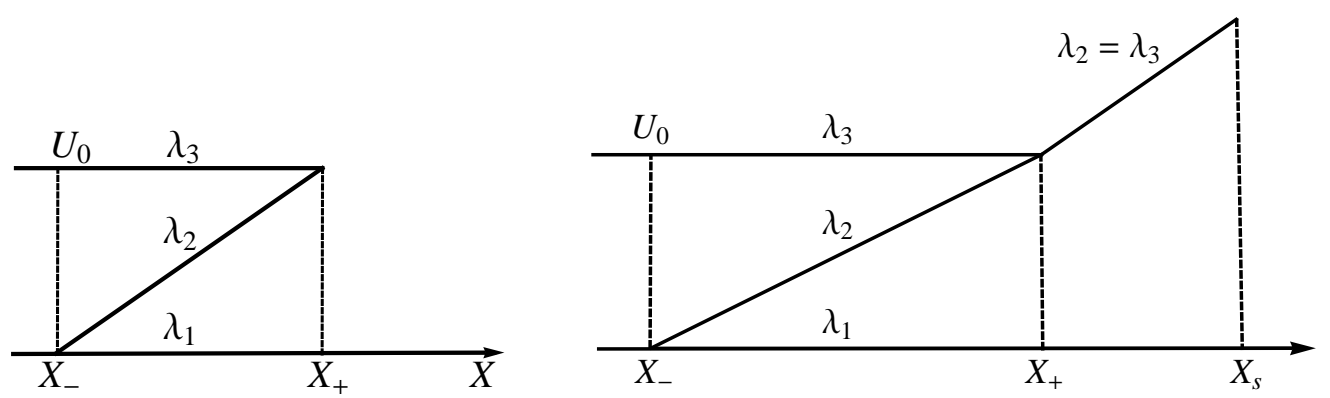

Figure 5. Schematic behaviour of the Riemann invariants in the modulation solution. Left: regular undular bore (before the slope, $T<T_{0}$ ); Right: undular bore with an advancing soliton train confined to $\left[X_{+}, X_{s}\right]$ (after the slope, $T \gg T_{1}$ )

$\lambda_{j}(X, T), j=1,2,3$ of the Whitham equations in the combined modulation solution obtained in Sections 3 and 4. These Riemann invariants $\lambda_{1} \leq \lambda_{2} \leq \lambda_{3}$ can be expressed as certain functions of any three independent modulation parameters from the set $\{a, q, V, d, m\}$ introduced in Section 3 but they are most conveniently represented in terms of the roots $b_{1} \leq b_{2} \leq b_{3}$ of the polynomial, defining the Riemann surface associated with the cnoidal wave periodic solution (3.1) (Whitham 1965), see formulae (5.4) in the next section. The periodic solution parameters $a, q, V$ and $d$ are expressed in terms of these Riemann invariants as

$a=2\left(\lambda_{2}-\lambda_{1}\right), \quad q=\sqrt{\lambda_{3}-\lambda_{1}}, \quad V=2\left(\lambda_{1}+\lambda_{2}+\lambda_{3}\right), \quad d=\lambda_{1}+\lambda_{2}-\lambda_{3}+4\left(\lambda_{3}-\lambda_{1}\right) \frac{E(m)}{K(m)}$.

Then the Gurevich-Pitaevskii modulation solution for the undular bore is given by $\lambda_{1}=0$, $\lambda_{3}=U_{0}$, while $\lambda_{2}=U_{0} m$ is defined by the same expression (3.4). In the solitary wavetrain we have $\lambda_{2}=\lambda_{3}$ to leading order so the asymptotic solution (4.23) as $T \rightarrow \infty$ assumes the form $\lambda_{3} \sim X /(4 T)$ in the interval $4 U_{0} T \leq X \leq 4 U_{0} T \beta_{1}^{-1 / 3}$.

The outlined behaviour of the Riemann invariants in the modulated solutions before 
and after the slope, is schematically shown in Fig 5 . This behaviour will be confirmed by direct numerical simulations in the next Section.

\section{Numerical simulations}

In this section we shall describe the numerical confirmation of the three main assumptions used in the modulation analysis of Sections 3 and 4: (i) the assumption that the undular bore on a slope can be described by a slowly modulated periodic solution of the KdV equation; (ii) the "weak interaction scenario" ensuring the behaviour of the leading solitary wave in the undular bore on a slope as an isolated KdV soliton; (iii) the assumption that the wave structure forming in front of the undular bore is indeed a solitary wavetrain (rather than part of a traditional "cnoidal" bore described by the standard Gurevich-Pitaevskii solution).

For the numerical simulations we shall be using the variable-coefficient $\mathrm{KdV}$ equation in the form (2.8),

$$
B_{\tau}+\frac{3}{2 h^{5 / 4}} B B_{X}+\frac{h}{6} B_{X X X}=0
$$

with the dependence $h(\tau)$ corresponding to the depth profile (3.9),

$$
h(\tau)=\left\{\begin{array}{lc}
1.0 & : \tau<400 \\
\left(1-\frac{\alpha(\tau-400)}{2}\right)^{2} & : 400<\tau<844.44 \quad, \quad \alpha=0.0009 \\
0.64 & : \tau>844.44
\end{array}\right.
$$

As was already mentioned, equation (5.1) is exactly equivalent to the equation (2.12) used for our asymptotic analysis. The present form (5.1) is slightly more convenient for numerical simulations as it produces fewer waves for the same running time interval. The initial condition is taken in the form of a smooth step,

$$
B(X, 0)=\frac{1}{4}(1-\tanh (X / 10))
$$


Equation (5.1) was solved using the method of lines where the spatial derivatives are discretised using second order accurate finite difference approximation to reduce the governing partial differential equation to a system of ordinary differential equations. This system is then solved using the fourth order Runge-Kutta method (see e.g. Schiesser (1991)).

Assuming that locally, undular bore can be described by the periodic solution (3.1) we shall use the numerical solutions to extract the parameters corresponding to the modulation Riemann invariants $\lambda_{j}$ introduced in (4.26), which are expressed in terms of the basic wave parameters $b_{1}, b_{2}$ and $b_{3}$ as (see Whitham (1965))

$$
\lambda_{3}=\frac{b_{2}+b_{3}}{2} \quad \lambda_{2}=\frac{b_{1}+b_{3}}{2} \quad \lambda_{1}=\frac{b_{1}+b_{2}}{2}
$$

Here $b_{2} \equiv B_{\min }$ and $b_{3} \equiv B_{\max }$ are easily found from the numerical data. The third parameter $b_{1}$ can be obtained from the numerical values of the local spatial period (wavelength) $L$, which for the variable-coefficient $\mathrm{KdV}$ equation (5.1) is given by the formula

$$
L=\frac{4 h^{9 / 8} K(m)}{\sqrt{3\left(b_{3}-b_{1}\right)}} \quad \text { where } \quad m=\frac{b_{3}-b_{2}}{b_{3}-b_{1}} .
$$

We expect that the variables $\lambda_{j}$ will demonstrate the qualitative behaviour shown in Figure 5 which will be a confirmation of the validity of the modulation analysis presented in Sections 3 and 4.

The results of our numerical simulations are presented in Figures 6 -10. Each of the Figures $6-9$ corresponds to a particular value of time, $\tau=\tau_{i}$ and consists of three plots: the top plot shows the numerical solution for $B\left(X, \tau_{i}\right)$, the middle plot shows spatial behaviour of the numerical "Riemann invariants" $\lambda_{j}$ and the bottom plot shows the behaviour of the numerically determined value of the modulus $m$.

Figure 6 corresponds to the initial undular bore, $\tau=400$, with the numerical values 
of the Riemann invariants $\lambda_{j}$ (the middle plot) distributed according to the GurevichPitaevskii solution (3.4) with $\beta=1$ and the modulus running from 0 to 1 across the bore (see the bottom plot). The Riemann invariant distribution agrees with the diagram shown on Fig. 5a.

Figure 7 corresponds to the bore propagating over the slope, $\tau=800$, and one can observe the presence of the region with $\lambda_{2}=\lambda_{3}$ in front of the bore. Since we have $m=1$ in this region (see the bottom plot), the corresponding wave structure should be identified with the solitary wavetrain. The amplitude $a=\lambda_{3}-\lambda_{1}$ in this solitary wavetrain increases towards the leading edge as predicted by our analysis (the comparison for the amplitude of the leading solitary wave is presented in Figure 10).

In Figure 8 we present the distributions corresponding to $\tau=1400$, i.e. "after" the slope. While the front part of the bore and the advancing solitary wavetrain retain their structure presented in Figure 7 one can observe the occurrence of the new multi-phase (presumably, two-phase) region in the rear part of the bore. As a matter of fact the modulus $m$ is not defined for this region (see the bottom plot).

Figure 9 shows the plots at $\tau=3400$ corresponding to the long-time behaviour of the transformed wavetrain. The distribution of the Riemann invariants agrees with diagram in Figure 5b, confirming our theoretical predictions. One can see that the behaviour of the upper envelope in the solitary wavetrain is markedly different from that in the undular bore behind it and agrees with the asymptotic formula (4.23).

Note the behaviour of the Riemann variables $\lambda_{2}$ and $\lambda_{3}$ near the leading edge of the undular bore in all plots. One can see that $\frac{d}{d X} \lambda_{2}<\infty$ and $\frac{d}{d X} \lambda_{3}<\infty$, which corresponds to the "weak interaction" scenario identified in El et al. (2007). This confirms our main assumption that the leading soliton in the undular bore behaves as an isolated solitary wave in the propagation over the decreasing depth region. The direct quantitative 
confirmation of this assumption can be seen in Figure 10 where we present the comparison for the amplitude of the adiabatically varying isolated solitary wave on a slope with the numerical values of the leading solitary wave amplitude in the modulated wavetrain (initially a single undular bore) propagating over the same slope. In both cases the initial value of the amplitude was the same, $a=1$. For the variable-coefficient KdV equation (5.1) the adiabatic variations of the solitary wave amplitude are given by formula (see

$$
a=a_{0}\left(\frac{h_{0}}{h(\tau)}\right)^{3 / 4},
$$

where $h_{0}$ and $a_{0}$ are the initial depth and the solitary wave amplitude respectively. Relationship (5.6) follows from (4.3) for the slowly varying solitary wave of the variablecoefficient $\mathrm{KdV}$ equation in the form (2.12). One can see an excellent agreement which provides direct quantitative confirmation our assumption that the leading solitary wave of the undular bore evolves as an isolated solitary wave over the variable depth region when $h^{\prime}(x)<0$.

In conclusion of this section we note that, although in our analysis it was assumed that the undular bore is fully developed by the moment it enters the slope region, this requirement is not a pre-requisite for the formation of the solitary wavetrain ahead of the undular bore. Indeed, our numerical simulations show that most of the key features of the shoaling undular bore transformation described above remain present in the case when the initial discontinuity is placed right at the beginning of the shoal so that the formation of the undular bore and the generation of the solitary wavetrain initially occur simultaneously, while the the whole structure is propagating over the slope. 

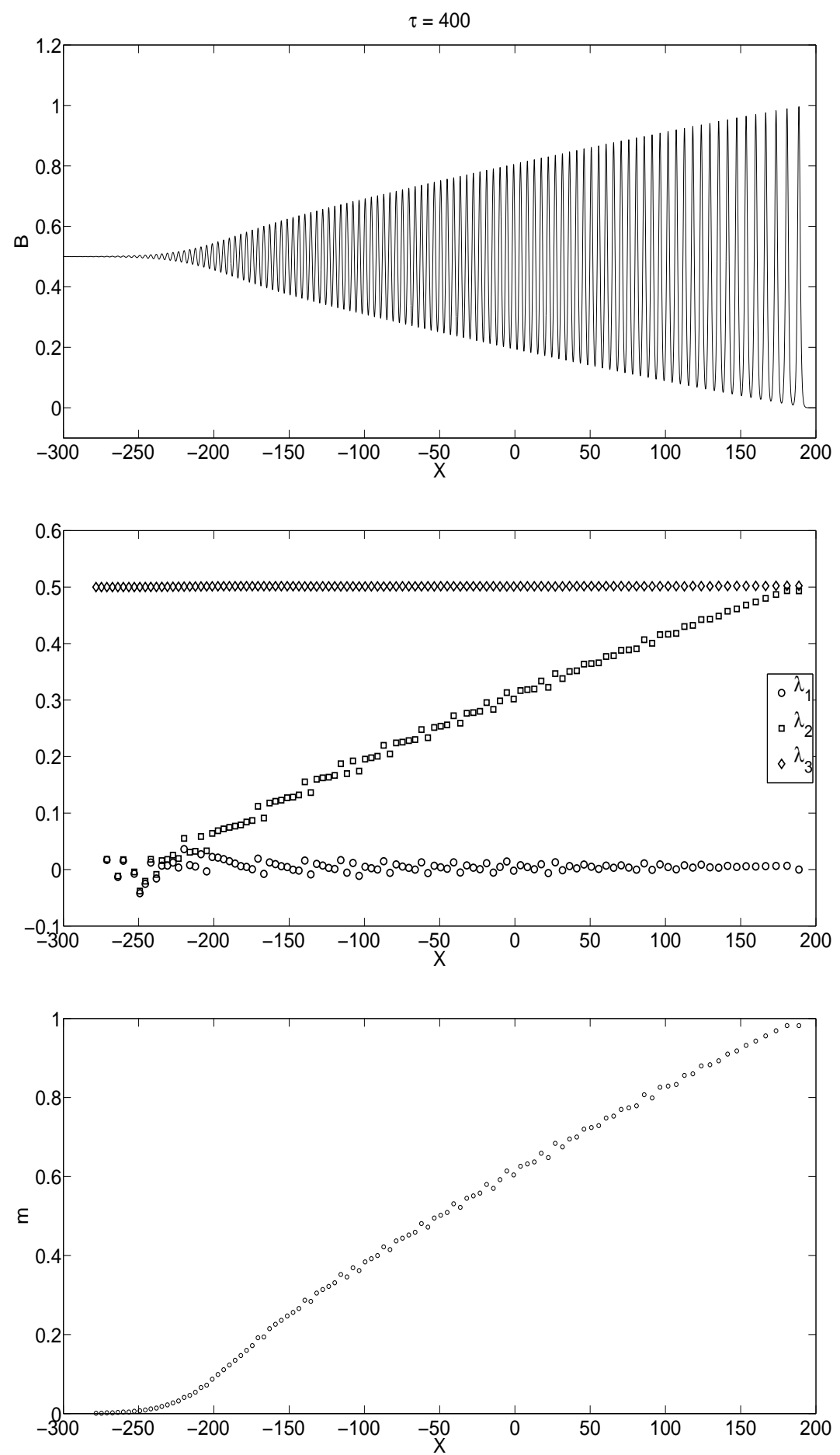

FIgURE 6. Initial undular bore (before slope), $\tau=400$. Upper plot: $B(X)$; Middle plot: the modulation Riemann variables $\lambda_{1} \leq \lambda_{2} \leq \lambda_{3}$ obtained from the plot for $B(X)$ assuming a local representation of the wave in the form of a cnoidal periodic solution of the constant-coefficient $\mathrm{KdV}$ equation; Bottom plot: the modulus $m=\left(\lambda_{2}-\lambda_{1}\right) /\left(\lambda_{3}-\lambda_{2}\right)$ as function of $X$. 

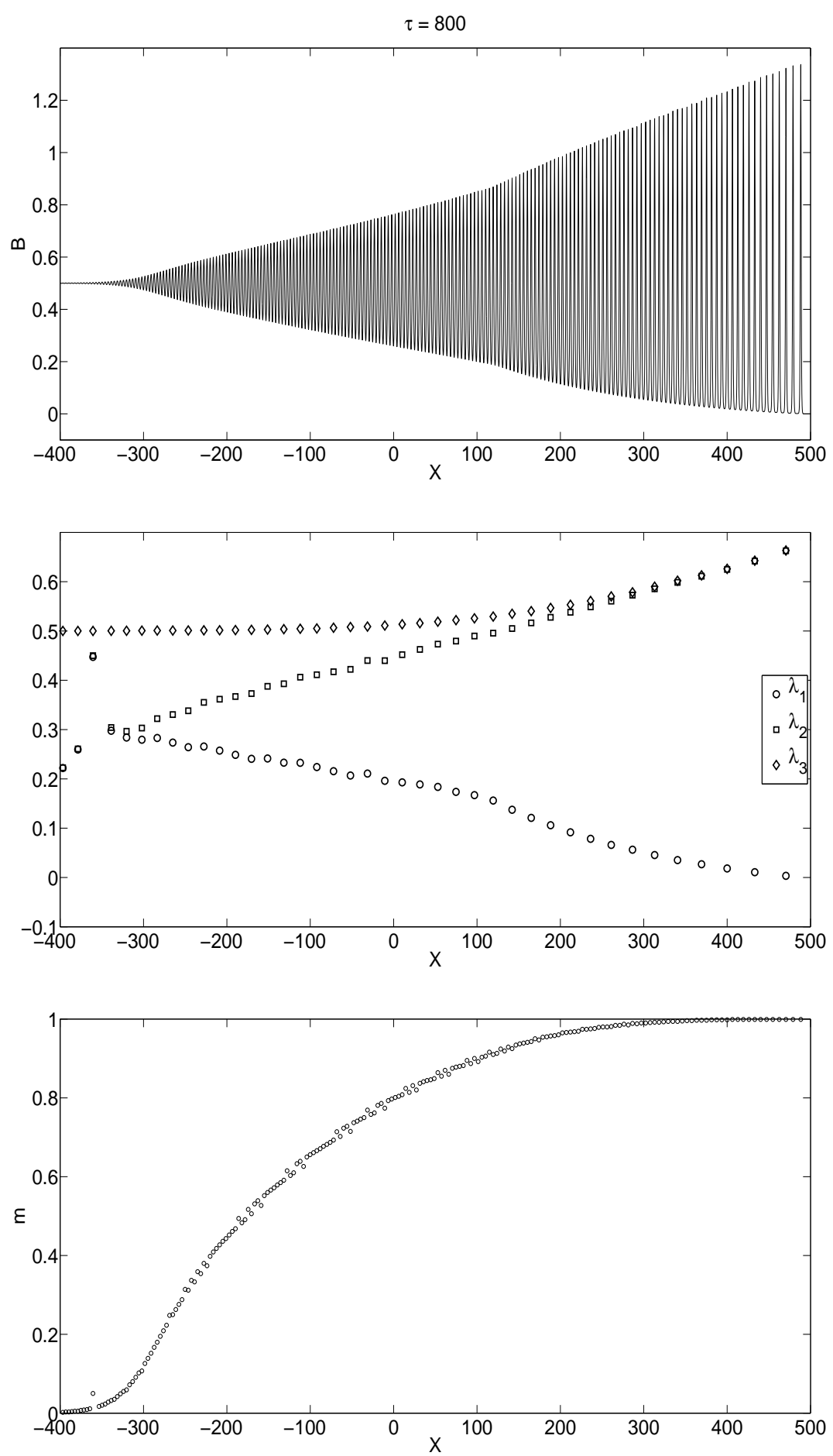

Figure 7. Same as in Figure 7 but for $\tau=800$ (undular bore on the slope). One can see the solitary wavetrain $(m \rightarrow 1)$ forming between $X \approx 350$ and $X \approx 500$. 

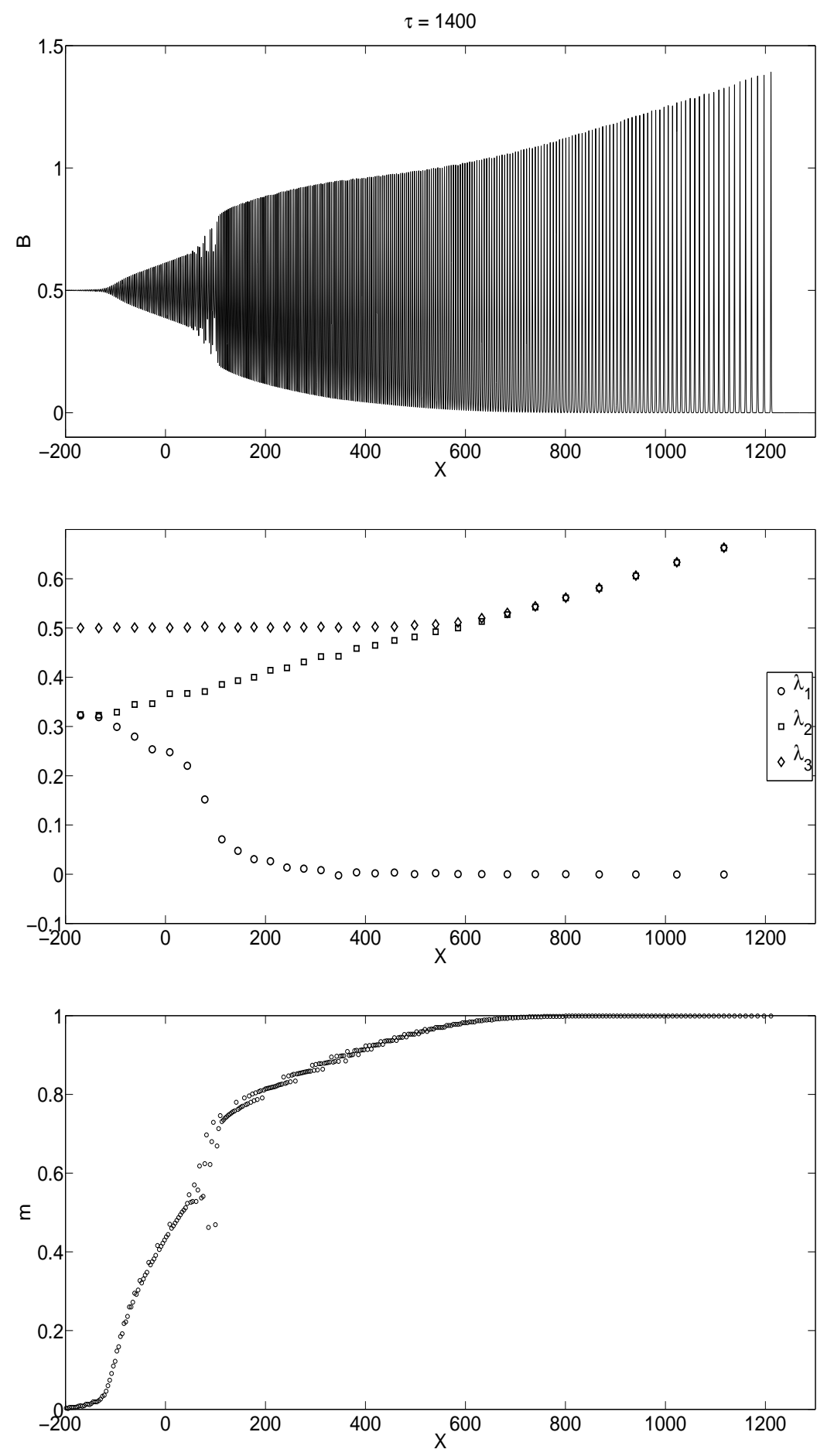

Figure 8. Same as in Figures 6,7 but for $\tau=1400$ (after the slope). The solitary wave train is located between $X \approx 700$ and $X \approx 1200$. A multiphase behaviour can be seen around $X=100$. 

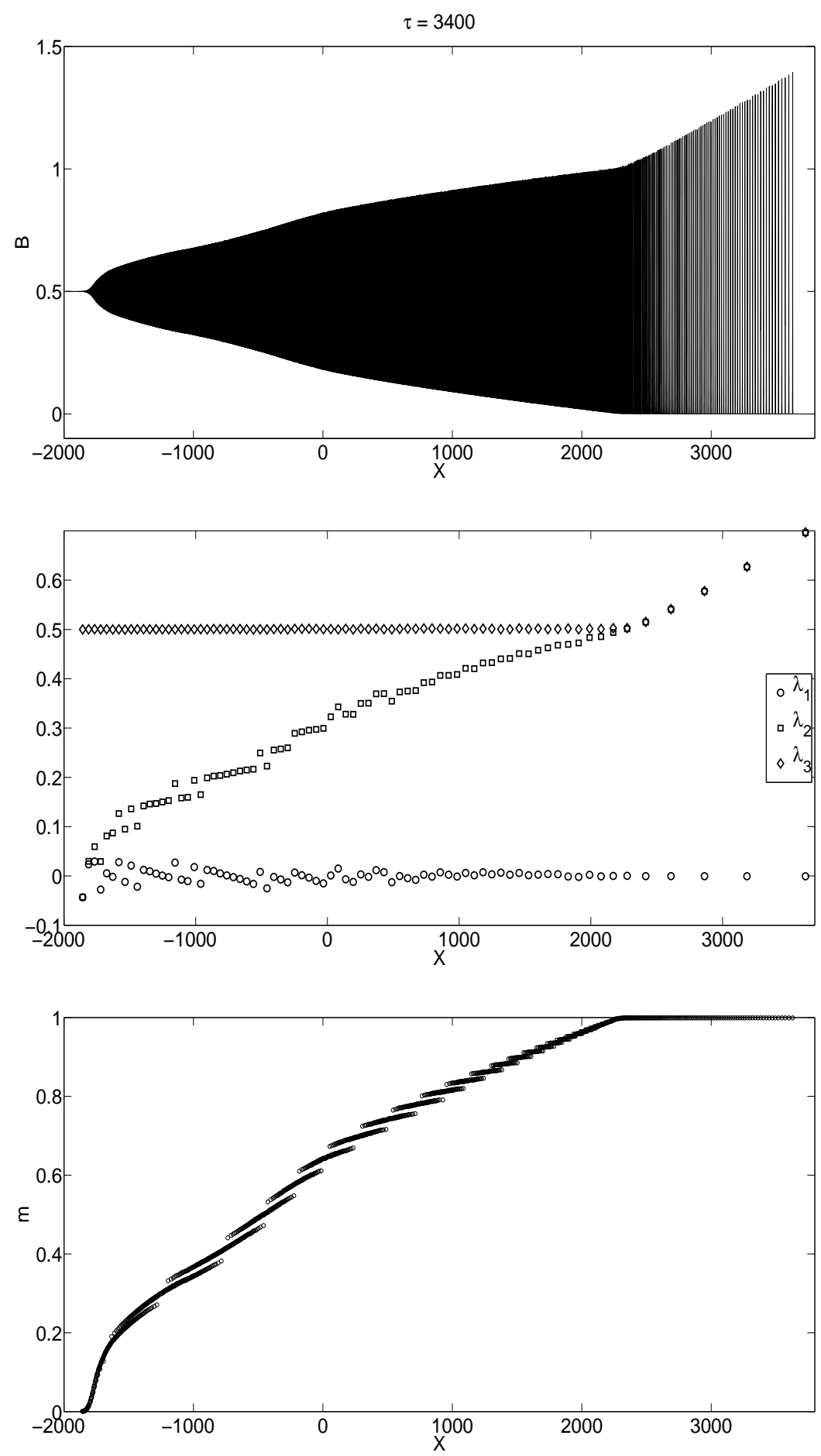

Figure 9. Same as in Figures 6,7,8 but for $\tau=3400$ (long-time behaviour after the slope). The linear behaviour of the envelope in the solitary wavetrain agrees with the asymptotic prediction (4.23). 


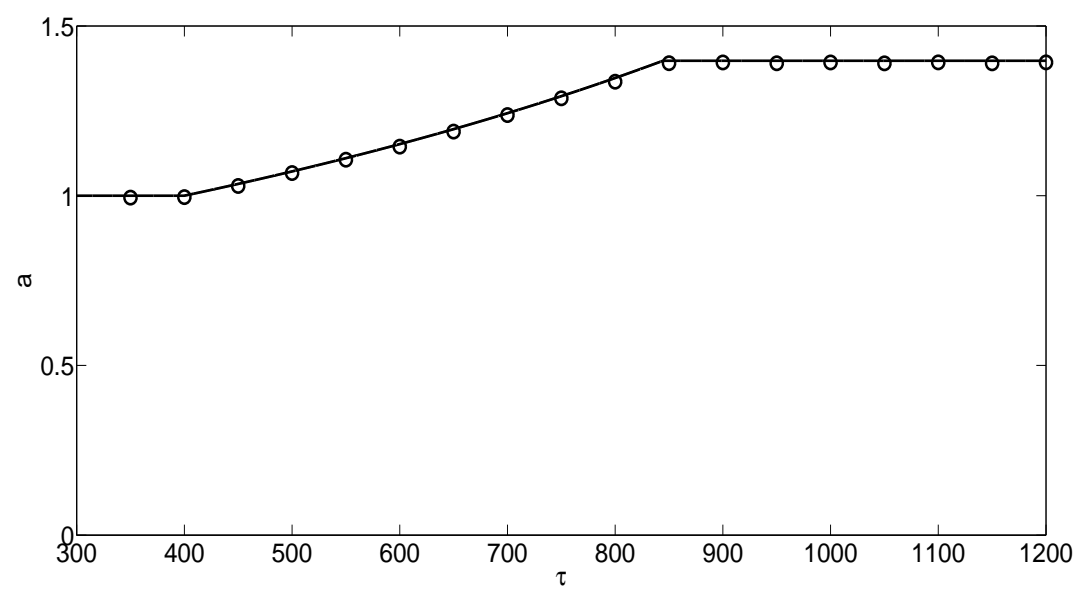

FIgURE 10. Comparison for the amplitude of the adiabatically varying solitary wave on a slope (formula (5.6) with $h_{0}=1, a_{0}=1$ ) - solid line; and the numerical data (circles) for the leading solitary wave in the modulated wavetrain (initially, a single undular bore on the same slope).

\section{Discussion}

In this paper, we have considered a shallow water undular bore propagation over a sloping bottom in the framework of the appropriate variable-coefficient KdV equation. We have shown that, when the undular bore advances into the decreasing depth region its interaction with the varying topography results in the formation of a sequence of isolated solitary waves, an expanding modulated solitary wavetrain propagating ahead of the bore and having the amplitude greater than that of the leading solitary wave in the undular bore. Unlike other mechanisms of the soliton train generation in the variable-coefficient KdV equation, such as soliton fission (Madsen \& Mei (1969), Johnson (1973)), soliton caustics (Malomed \& Shrira (1991)) or the formation of secondary solitons in the trailing shelves (Kaup \& Newell (1979), Kivshar \& Malomed (1989), El \& Grimshaw (2002)), the presented new mechanism is essentially related to non-adiabatic deformations of modulated cnoidal waves rather than transformations/decay of individual solitons.

Using several assumptions (confirmed by detailed numerical simulations) about the 
structure of the undular bore propagating on a slope we have constructed an asymptotic modulation solution describing the generation and propagation of the solitary wavetrain ahead of the undular bore. Our analysis can be extended to include the effects of small dissipation (e.g. turbulent bottom friction).

Importantly, our general approach is not confined to the $\mathrm{KdV}$ dynamics and can be applied to other systems describing the propagation of undular bores (or, more generally, dispersive shock waves) through weakly non-uniform environments. In particular, it could be used for the description of the generation of solitary wavetrains by internal undular bores in the ocean where the waves typically are propagating on a background whose properties vary in the wave propagation direction. The relevant model here is an extended $\mathrm{KdV}$ (Gardner) equation which is often used to model oceanic internal solitary waves over bottom shelves (see e.g. Grimshaw et al. (2004)). We also stress that the availability of the full modulation solution (an analog of the Gurevich-Pitaevskii solution (3.4)) for the initial "flat-bottom" undular bore is not a pre-requisite in our analysis, and a similar study can be undertaken for the systems where the initial evolution of the undular bore is described by a non-integrable dispersive equation (see El (2005) for the relevant generalisation of the Gurevich-Pitaevskii problem). In particular, one can consider the propagation of a fully nonlinear shallow water undular bore over a slope in the framework of the appropriate variable-coefficient Su-Gardner (Green-Naghdi, or Serre) equations (see El et al. (2006) for the corresponding flat-bottom undular bore theory).

\section{Appendix: Determination of $\kappa_{0}$}

We assume here that function $\beta(T)$ is sufficiently slow so that for each $T$ the undular bore can be locally described by a single-phase solution (3.1). As our numerical simulations show, this is not entirely true for the whole wavetrain but definitely holds for the 
front part of the bore. The wavenumber in the bore is given by the formula (3.6),

$$
k=\frac{\pi U_{0}^{1 / 2}}{\beta^{1 / 2} K(m)} .
$$

If we consider the solitary wavetrain as a small wavenumber asymptotic of the modulated cnoidal wave we must have (see Whitham (1974))

$$
1-m \ll 1: \quad \kappa \simeq \frac{k}{2 \pi},
$$

which suggests a continuous matching between $k /(2 \pi)$ in the undular bore and $\kappa=\kappa_{0}$ in the solitary wavetrain at the leading edge $X_{+}(T)$ of the undular bore. This continuous matching, however, is not possible for the following reason.

The asymptotic behaviour of $k$ in the modulation solution (3.4) near the leading edge $X=X_{+}(T)$ is (Gurevich \& Pitaevskii (1974))

$$
k \sim \frac{2 \pi}{\beta^{1 / 2}} \frac{U_{0}^{1 / 2}}{\ln \left(1 /\left(s_{+}-s\right)\right)},
$$

where $s=X / T, s_{+}=X_{+} / T=4 U_{0}$. This implies that $\left|k_{X}\right| \rightarrow \infty$ while $k \rightarrow 0$ when $X \rightarrow X_{+}$. Thus the wavenumber varies rapidly near $X=X_{+}$. On the other hand, the typical spatial scale of the variations of modulations in the advancing solitary wavetrain is much greater than that in the undular bore. Therefore it is natural to require matching of $\kappa$ with the mean value of $k /(2 \pi)$ across the undular bore front, which one can naturally define as the (soliton) part of the bore propagating to the right, i.e. $0<X<X_{+}$(note that $X=0$ is the characteristic of the modulation system separating the "rightward and leftwards propagating" parts of the characteristic fan (3.4)). The mean value of $k$ across the bore front is then

$$
\bar{k}=\frac{1}{X_{+}} \int_{0}^{X_{+}} k d X=\frac{1}{4 U_{0}} \int_{m^{*}}^{1} k(m) \cdot\left(\frac{d s}{d m}\right) d m,
$$

where $s(m)$ is given by the modulation solution $(3.4)$ and $m^{*} \approx 0.6415$ - the value of $m$ on the characteristic $X=0-$ is the root of the equation $s(m)=0$. Then, using (6.1) 
we obtain

$$
\bar{k}=\frac{\pi U_{0}^{1 / 2}}{2 \beta^{1 / 2}} I,
$$

where

$$
\begin{gathered}
I=\int_{m^{*}}^{1} \frac{W^{\prime}(m)}{K(m)} d m \approx 0.6569, \\
W(m)=1+m-\frac{2 m(1-m) K(m)}{E(m)-(1-m) K(m)} .
\end{gathered}
$$

Thus we have

$$
\kappa_{0}=\frac{\bar{k}}{2 \pi}=\frac{U_{0}^{1 / 2}}{4 \beta^{1 / 2}} I
$$

\section{REFERENCES}

Ablowitz, M.J., Baldwin, D.E. \& Hoefer, M.A. 2009 Soliton generation and multiple phases in dispersive shock and rarefaction wave interaction. Phys. Rev. E 80, 016603.

BAines, P.G. 1995 Topographic effects in stratified flows. Cambridge University Press.

BoussinesQ, J. 1872 Théorie des ondes des remous qui se propagent le long d'un canal rectangulaire, en communuuant au liquide contenu dans ce canal des vitesses sensblemnt pareilles de la surface au fond. J. Math. Pures Appl. 17, 55-108.

Claeys, T. \& Grava, T. 2010 Solitonic asymptotics for the Korteweg-de Vries equation in the small dispersion limit. SIAM J. Math. Analysis 42, 2132-2154.

EL, G.A. \& Grimshaw, R.H.J. 2002 Generation of undular bores in the shelves of slowlyvarying solitary waves. Chaos 12, $1015-1026$.

El, G.A., R.H.J., Grimshaw \& Kamchatnov, A.M. 2007 Evolution of solitary waves and undular bores in shallow-water flows over a gradual slope with bottom friction. Journ. Fluid Mech. 585, 213-244.

EL, G. A. 2005 Resolution of a shock in hyperbolic systems modified by weak dispersion. Chaos 15, 037103 .

El, G. A., Grimshaw, R. H. J. \& Smyth, N. F. 2006 Unsteady undular bores in fully nonlinear shallow-water theory. Phys. Fluids 18, 027104. 
El, G. A., Grimshaw, R. H. J. \& Smyth, N. F. 2009 Transcritical shallow-water flow past topography: finite-amplitude theory. Journ. Fluid Mech. 640, $187-215$.

EL, G. A., Khodorovskit, V. V. \& Leszczyszyn, A. M. 2011 Refraction of dispersive shock waves. arXiv:1105.1920, submitted to Physica D .

Esler, J. G. \& Pearce, J. D . 2011 Dispersive dam-break and lock-exchange flows in a two-layer fluid. Journ Fluid Mech 667, 555-585.

Fornberg, B. \& Whitham, G. B. 1978 A numerical and theoretical study of certain nonlinear wave phenomena. Philosophical Transactions of the Royal Society A 289, 373-404.

GrimshaW, R. 1979 Slowly varying solitary waves. I Korteweg-de Vries equation. Proc. Roy. Soc. 368A, 359-375.

Grimshaw, R. 1981 Evolution equations for long nonlinear internal waves in stratified shear flows. Stud. Appl. Math. 65, 159-188.

Grimshaw, R. 2007a Internal solitary waves in a variable medium. Gesellschaft für Angewandte Mathematik 30, 96-109.

Grimshaw, R. $2007 b$ Solitary waves propagating over variable topography. In Tsunami and Nonlinear Waves (ed. A. Kundu), pp. 49-62. Springer.

Grimshaw, R., Pelinovsky, E., T. Talipova, T. \& Kurkin, A. 2004 Simulation of the transformation of internal solitary waves on oceanic shelves. J. Phys. Ocean. 34, 27742779 .

Grimshaw, R. H. J. \& Smyth, N. F. 1986 Resonant flow of a stratified fluid over topography. J. Fluid Mech. 169, 429-464.

Gurevich, A. V. \& Pitaevskit, L. P. 1974 Nonstationary structure of a collisionless shock wave. Sov. Phys. JETP 38, 291-297.

Johnson, R.S. 1997 A modern introduction to the mathematical theory of water waves. Cambridge University Press.

Johnson, R. S. 1973 On the development of a solitary wave moving over an uneven bottom. Proc. Camb. Phil. Soc. 73, 183-203.

Kakutani, T. 1971 Effect of an uneven bottom on gravity waves. J. Phys. Soc. Japan. 30, $272-276$. 
Kamchatnov, A.M. 2004 On Whitham theory for perturbed integrable equations. Physica D188, 247-261.

Kaup, D.J. \& Newell, A.C. 1979 Solitons as particles, oscillators, and in slowly changing media : a singular perturbation theory. Proc. Roy. Soc. London A 361, 413-446.

Kawahara, T. 1975 Derivative-expansion method for nonlinear waves on a liquid layer of slowly varying depth. J. Phys. Soc. Japan 38, 1200-1206.

Khruslov, E. A. 1976 Asymptotics of the solution of the Cauchy problem for the Korteweg-de Vries equation with step-like initial data. Math. USSR-Sb. 28, 229-248.

Kivshar, Yu.S. \& Malomed, B.A. 1989 Dynamics of solitons in nearly integrable systems. Rev. Mod. Phys. 61, 763-907.

Madsen, O.S. \& Mei, C.C. 1969 The transformation of a solitary wave over an uneven bottom. J. Fluid Mech. 39, 781-891.

Madsen, P. A., Fuhrman, D. R. \& Schäffer, H. A. 2008 On the solitary wave paradigm for tsunamis. J. Geophys. Res. 113, C12012.

Malomed, B.A. \& Shrira, V.I. 1991 Soliton caustics. Physica D53, 1-12.

Miles, J.W. 1983a Solitary wave evolution over a gradual slope with turbulent friction. J. Phys. Oceanog. 13, 551-553.

Miles, J.W. $1983 b$ Wave evolution over a gradual slope with turbulent friction. J. Fluid Mech. 133, 207-216.

Newell, A. 1985 Solitons in mathematics and physics. SIAM, Philadelphia, PA.

Ostrovsky, L.A. \& Pelinovsky, E.N. 1975 Refraction of nonlinear sea waves in a coastal zone. Akad. Nauk SSSR, Izv. Atmos. Ocean Phys. 11, 37-41.

SCHIESSER, W. E. 1991 The numerical method of lines: integration of partial differential equations. Academic Press.

Scotti, A., Beardsley, R.C., Butman, B. \& Pineda, J. 2008 Shoaling of nonlinear internal waves in Massachusetts bay. J. Geophys. Res. 113, C08031.

Smyth, N.F. \& Holloway, P.E. 1988 Hydraulic jump and undular bore formation on a shelf break. J. Phys. Oceanog. 18, 947-962.

Tissier, M., Bonneton, P., Marche, F., Chazel, F., \& Lannes, D. 2011 Nearshore dy- 
namics of tsunami-like undular bores using a fully nonlinear Boussinesq model. Journal of Coastal Research Special Issue 64, 603-607.

Whitham, G.B. 1965 Non-linear dispersive waves. Proc. Roy. Soc. London A 283, 238-261.

Whitham, G.B. 1984 Comments on periodic waves and solitons. IMA Journ. Appl. Math. 32, 353-366.

Whithaм, G. B. 1974 Linear and Nonlinear Waves. J. Wiley and Sons. 